\title{
P40
}

\section{Geomechanically-derived Fault Zone Petrophysical Properties - A Synthetic Fault Model Study}

\author{
A. Paez (Heriot-Watt University), H. Lewis* (Heriot-Watt University), G.D. \\ Couples (Heriot-Watt University) \& J. Ma (Heriot-Watt University)
}

\section{SUMMARY}

A 2D geomechanical model of a fault zone has been used to develop porosity and permeability distributions that reflect the geomechanical rock damage. A pseudo-3D geomodel, where fault displacment varies from zero to maximum has been created and a method developed to appropriately populate it with porosity and permeability values. Both dilational and compactional deformation occurs in this normal fault,as is expected in real subsurface faults, resulting in both increased and decreased (relative to the matrix) permeability values. Geometrically equivalent geomodels using a range of transmissibility multiplier to represent the fault have also been generated. These six geomodels ( 5 with transmissibity multipliers and 1 with geomechanically-derived poroperm) have then been converted into an industrystandard flow simulation that vary only in the treatment of petrophysical properties around the fault zone. The resulting flow simulations show that the geomechanically-controlled poroperm properties to a better job of representing the kind of complexity of e.g. across-fault pressure regimes and fluid saturations that are encountered in subsurface reservoirs. 


\section{Fault and Top Seals \\ Montpellier 2009}

Natural fault zones are widely recognised as having considerable evolved character in their deformation fabrics. These fabrics are very far from uniform or uniformly varying with, for example, fault displacement or burial depth. Instead they develop distinct patterns over a range of length scales. The different component parts of the evolved fabric also have distinctly different petrophysical characteristics which can themselves be very different from those of the host rock. However fluid flow simulations tend to treat the permeability across fault zones as a constant expressed as a transmissibility multiplier (Tm). Geomechanics is seldom included in such simulations, but when it is included, it is typically used to modify the stress state around the fault zone and does not address the changed, and spatially variable, poroperm properties.

This work explores the potential to use realistic (look-alike) deformation fabrics that have been generated by geomechanical simulations of fault zone evolution which specifically incorporate poroplastic behaviour and therefore can develop deformation-induced permanent volume increase/loss (Couples et al 2007, Lewis et al, 2007). The resulting volumetric strain fields are then converted into porosity and permeability distributions using a simple poroperm transform. These distributions are in turn used in industry-standard 2-phase flow simulations and the results are compared to otherwise identical models for which the fault zones are treated as having one of a series of transmissibility multipliers.

The geomechanical simulations used in this study are of a geometrically simple normal fault in a single layer of limestone. It is 2D in the vertical plane. The model is constrained such that the rock package achieves an approximately uniformly $60^{\circ}$ dipping fault (figure 1 ) and fault displacement is increased in small uniform displacement steps. Figure 1 shows the central part of that geomechanical model at maximum fault displacement.
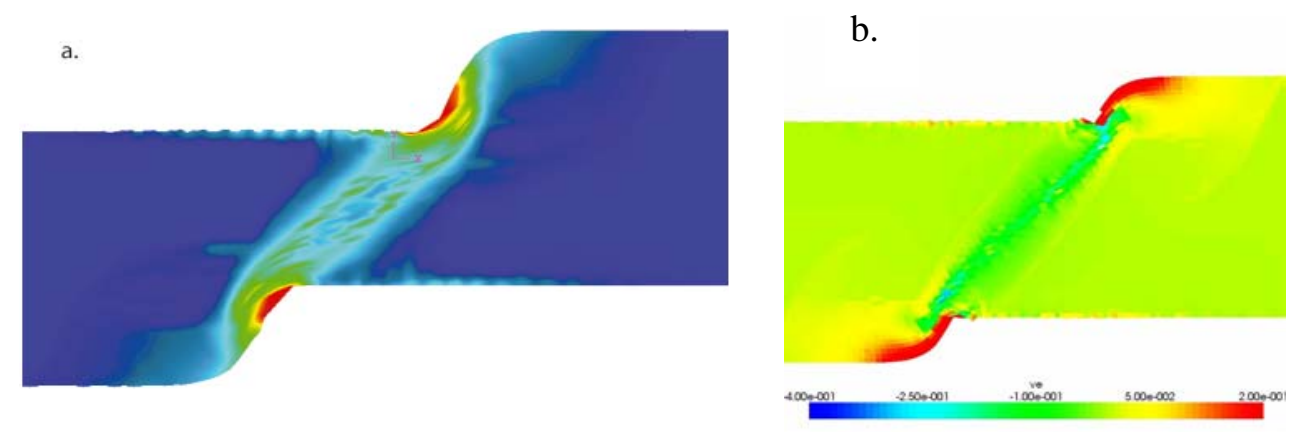

Figure 1. 2D geomechanical model of a $60^{\circ}$ normal fault developed in a single limestone layer $50 \mathrm{~m}$ thick using a poroplastic material law (a.) Effective plastic strain magnitude, where blue is elastic only and red is $120 \%$. After Lewis et al. 2009. (b.) Volumetric strain where red and yellow are dilational and turquoise and blue are compactional.

The 2D geomechanical model of the limestone layer has been converted into a pseudo-3D model by stacking the displacement increments, creating a 3D normal fault with variation in throw (fig. 2). A series of six pseudo-3D models are then created, 5 having different Tms ranging from zero (completely sealed) to 1 (completely open). The sixth model has no Tm but instead using geomechanically-derived poroperm properties, assigned cell by cell. The Tms for models 1 through 5 are: 0 (model 1); 0.001 (model 2); 0.01 (model 3); 0.1 (model 4) and 1.0 (model 5). For the $6^{\text {th }}$, geomechanical, model the fault properties are calculated as a function of volumetric strain $\left(e_{\mathrm{v}}\right)$. Permeability and porosity distributions of the deformed rock reflect both enhancements and reductions within the damage zone.

The fluid flow simulations used a top-reservoir burial depth of $1 \mathrm{~km}$. with an initial fluid pressure of 150 bars, a porosity of $18 \%$ and a permeability of $1500 \mathrm{mD}$. The oil-water-contact was assumed to be at $1500 \mathrm{~m}$ depth and Pc was set to zero. All six cases are simulated using a two phase oil-water system. A single relative permeability curve is used for the entire mode. 


\section{Fault and Top Seals \\ Montpellier 2009}

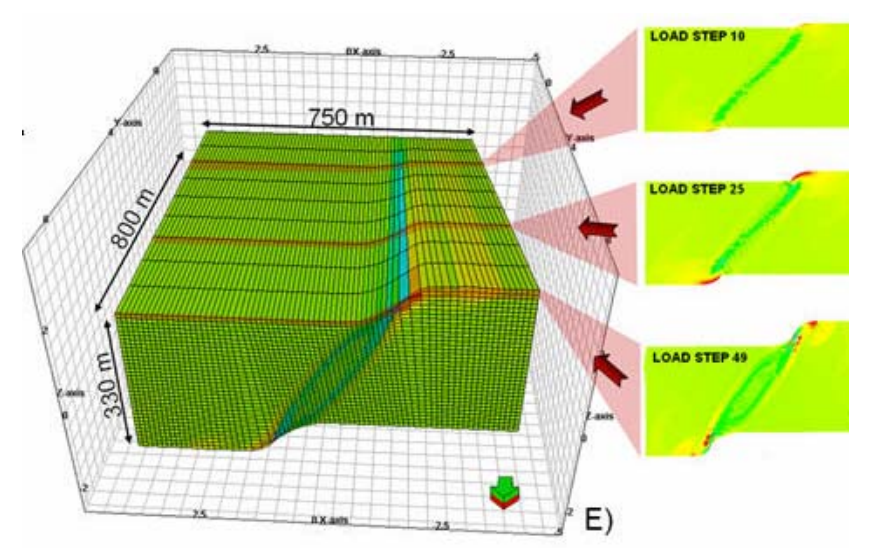

Figure 2. Pseudo-3D geomodel of normal fault with zero fault displacement at back on maximum displacement (step 50) at front. Colour shows magnitude of effective plastic strain. After Paez et al., 2008.

The Eclipse ${ }^{\circledR}$ default black oil fluid properties are used and production rate is set at 150 Sm3/day. The same injector and producer well distribution is used.

The six suites of flow simulations show, unsurprisingly, that the five geomodels with different transmissibility multipliers show different results, but the role the fault zone plays in each of these models is quite simple. For example, flow rates are degraded as compared to the rates in the unfaulted regions. In contrast, the geomechanically-derived geomodel (6) shows increased permeability regions in and around the faults zone as well as having degraded regions. Final pressure distributions, and fluid (oil and water) saturations (fig. 3) in all six models also reflect this separation into relatively simple behaviour where a transmissibility multiplier has been used and more complex, and potentially more realistic, where the fault zone properties have been derived from a geomechanical simulation which develops evolved rock properties.
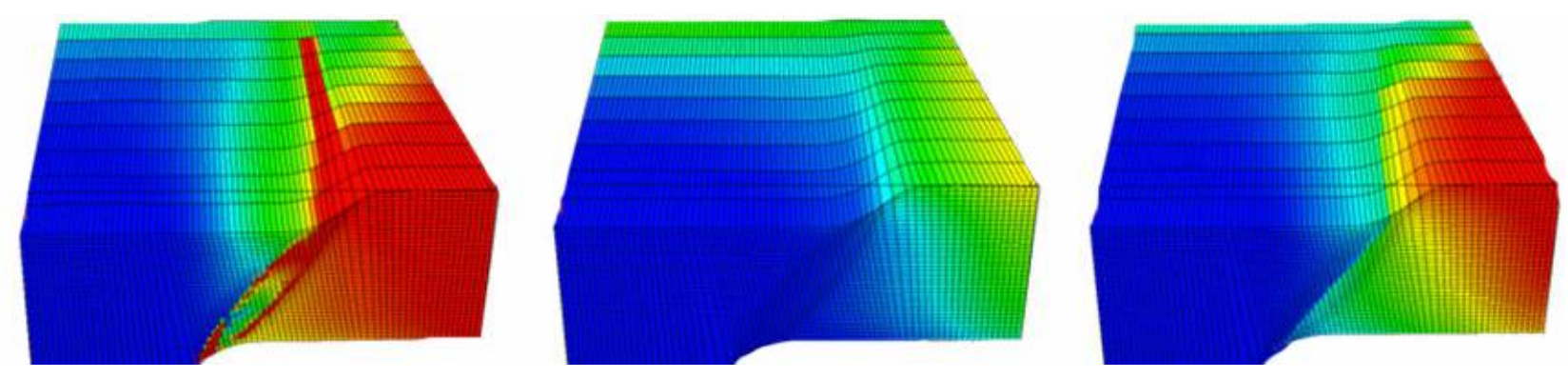

Figure 3. Two-phase flow simulations across simulated fault zone. Red is oil, blue is water. Left image is model 6 , with geomechanically-derived properties. Middle and right images use constant transmissibility multipliers of 0.1 and 0.01 respectively.

References:

Couples, G.D. Lewis, H., Olden, P., Higgs, N, and Workman, G. 2007. New insights into the faulting process using numerical simulations of rock-layer bending. In Lewis, H. \& Couples,G.D. (eds) 2007. The Relationship between Damage and Localization. Geological Society, London, Special Publications, 289, 161-186.

Lewis, H. and Hall, S. H, 2009. Geomechanical simulation to predict open subsurface fractures. Geophysical Prospecting, v.57, p. 289-295.

Lewis, H., Hall, S.A., Guest, J. \& Couples, G.D. 2007. Kinematically-Informed Geomechanical Simulations of Structural Evolution: Importance of Correct Loading for Open Fracture Prediction. In: Jolley, S., Barr, D., Knipe, R., and Walsh, J (Eds) Structurally Complex Reservoirs, Geological Society of London, Special Publications, 291, pp 159-172. Paez, A., Lewis, H., Ma, J. and Couples. G.D. 2008. Three dimensional distribution of geomechanically based fault properties and their consequences for poroperm and overpressure: a synthetic fault model study. AAPG, Cape Town South Africa . 